“ (C) 2016 IEEE. Personal use of this material is permitted. Permission from IEEE must be obtained for all other uses, in any current or future media, including

reprinting/republishing this material for advertising or promotional purposes, creating new collective works, for resale or redistribution to servers or lists, or reuse of any copyrighted component of this work in other works." 


\title{
SNR Threshold for Distributed Antenna Systems in Cloud Radio Access Networks
}

\author{
Ying He, Eryk Dutkiewicz, Gengfa Fang \\ Department of Engineering \\ Macquarie University, Australia \\ E-mail: \{y.he, eryk.dutkiewicz, gengfa.fang\}@mq.edu.au
}

\author{
Markus Dominik Mueck \\ Intel Mobile Communications, Germany \\ Email: markus.dominik.mueck@intel.com
}

\begin{abstract}
A distributed antenna system (DAS) architecture is a key enabler for Cloud Radio Access Networks (CRAN) where geographically separated base stations are connected to a centralized processing and decision making unit. Many schemes have been proposed to leverage Fractional Frequency Reuse (FFR) and co-ordinated joint transmission between base stations to improve cell-edge performance for static network deployments. In this paper, we investigate dynamic decision making that whether co-ordinated joint transmission should be selected in the downlink of a FFR-aided DAS. We derive the transmitting Signal-to-Noise-Ratio (SNR) threshold that co-ordinated joint transmission can provide better performance if the transmitting SNR is below this threshold. We simulated a three-cell cluster layout with FFR and the numerical results agree with our analytical results. We show that the transmitting SNR threshold is critical in the FFR-aided DAS analysis and can be used as a guide in the CRAN network planning and the evaluation of DAS performance.
\end{abstract}

Index Terms-Distributed Antenna Systems, Fractional Frequency Reuse, Coordinated Multi-Point Joint Transmission, Cloud Radio Access Networks.

\section{INTRODUCTION}

A Distributed Antenna System (DAS) is regarded as one of the promising methodologies for the next generation wireless communication, especially for the cellular networks. The concept of DAS was proposed in [1] and applications have been used in indoor systems. Subsequently, the concept was introduced into the cellular network by deploying multiple antennas in different sectors to improve performance within one cell or one macro cell. Recent research shows that DAS has the potential in a larger range of cellular networks [2],[3].

Recently, there has been growing interest in the area of Cloud Radio Access Networks (C-RAN) [4]which is an efficient and flexible architecture for future cellular networks. All signal processing and computing will be performed in a central unit, such as a super base station. Previous existing base stations become Remote Radio Frequency Units connected to the central unit through fiber with an RF switch. Since they are geographically separated, they can be employed as antennas in DAS in the cellular network. These features make efficient cooperation between cells possible and show the potential in improving cell edge users performance.

Most State-of-the-Art in this area (e.g. [5], [6]), has explored DAS performance with Coordinated Multi-Point (CoMP) and FFR. [5] proposed a frequency allocation scheme for CoMP

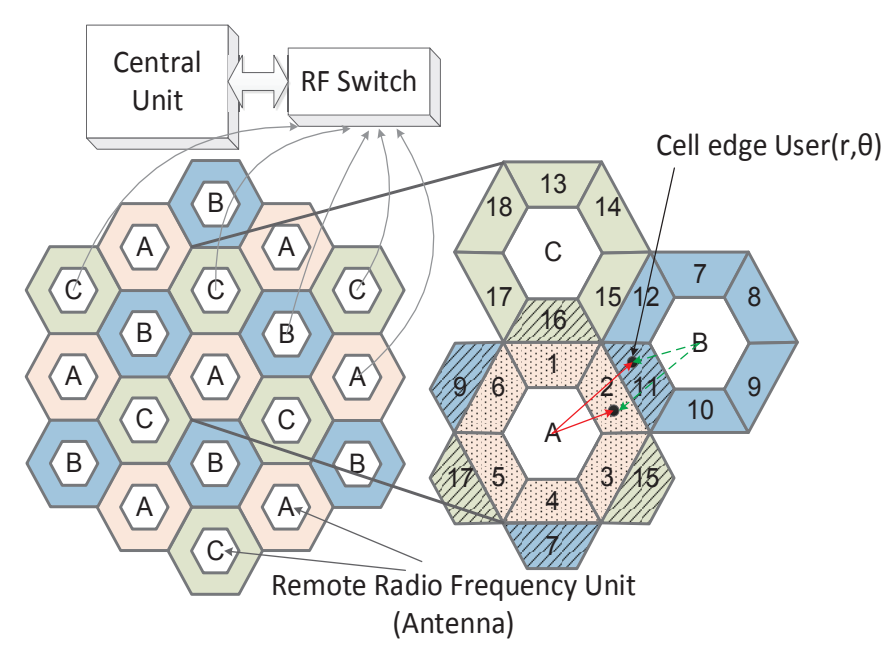

Fig. 1: Cloud Radio Access Networks Architecture with Fractional Frequency Reuse.

joint transmission with omni-directional and 6-sector directional antenna which can improve spectral efficiency for cell boundary users. [6] studied the unity frequency reuse and FFR in DAS. These above mentioned papers already proved that FFR has better performance than universal frequency reuse. Yet, none of these mentioned papers has considered requirements for dynamic set-ups of cells nor the effect of the transmitting SNR on Inter-cell Interference (ICI). These papers selected the thermal noise as the noise level in their simulation, which set their analysis to very high transmitting SNR cases. In [7], the authors have proved that DAS capacity may perform differently under high and low transmitting SNR levels and for cell-edge users the noise power is not low enough to be ignored. Thus, the problem of co-ordinated joint transmission in FFR-aided DAS for cell edge users has not been fully investigated.

In this paper, we derive an SNR threshold for co-ordinated and non-co-ordinated joint transmission. To the best of our knowledge, this is the first work that derives transmitting SNR threshold for FFR-aided co-ordinated and non-co-ordinated joint transmission in CRAN. The SNR threshold can provide a design guide for basestation co-ordinated transmission scheme 
in network planning without the need of simulations.

Notation: $E[\cdot]$ denotes the expectation operator.

\section{SySTEM MOdEL}

Consider a C-RAN multicellular and multiuser system including hexagonal shaped cells with one antenna in each cell and single-antenna users in each cell. All the antennas are geographically distributed and connected through fiber to a remote central unit, as illustrated in Fig. 1.

Assume that users are uniformly distributed in each cell, the user number is the same for each cell and for each user there is another user occupying the same frequency in the interfering cell. For cell-edge users them can use up to $B W_{\text {edge }}=B W_{\text {all }}-B W_{\text {center }}$ bandwidth, where $B W_{\text {all }}$ is the total bandwidth, $B W_{\text {edge }}$ is the bandwidth in the cell edge and $B W_{\text {center }}$ is the bandwidth in the cell center. Assume that the size of the cell center is $2 / 3$ of the cell radius [7].

The channel assumption is that the channel from each base station is independent from the others and the transmitting power is $P_{t}$. The cell deployment is a two-tier 19-cell model in this paper. The antennas are the ones with shortest distances to the user among all antennas. We consider two transmitting schemes in this paper: co-ordinated joint transmission (CJT) and non-co-ordinated joint transmission (non-CJT). CJT represents multiple base stations jointly transmit to a user with one antenna at each base station. Non-CJT represents one base station transmit to a user with one antenna at each base station. We assume that the cells apply either all CJT or all non-CJT. The downlink receiving signal by a user $k$ is given by:

$$
y_{k}=\sum_{j \in B_{S}} \sqrt{P_{t}} h_{j k} x_{k}+\sum_{i \in B_{I}} \sqrt{P_{t}} h_{i k^{\prime}} x_{i k^{\prime}}+n_{k}
$$

where $x_{k}$ and $x_{i k^{\prime}}$ are the transmitting signal of the reference user and the interfering users; $n_{k}$ is the Additive White Gaussian Noise (AWGN) whose power density is $N_{0}$.

The transmitting antenna set is $B_{S}$ and the interfering antenna set is $B_{I},\left|B_{S}\right|$ is the number of antennas in joint transmission and $\left|B_{I}\right|$ is the number of interfering antennas. We divided each cell into 6 sectors, each sector will be allocated a certain equal frequency resources. We use threecell cluster cellular topology. In one cluster, illustrated as cell $\mathrm{A}, \mathrm{B}$ and $\mathrm{C}$, there are 18 different sectors, labeled as $1, \ldots, 18$ in Fig. 1. If any sector in the overall 19 cells uses the same frequency, the respective cell is considered as having a interfering basestation [8].

In this three-cell cluster model, the co-ordinated basestation number is at most three, because more than three basestations' co-ordination will cause interference inside of a cluster. For CJT transmission, the user is supported by two or three antennas, $\left|B_{S}\right|=2$ or 3; for non-CJT transmission, the user is only supported by one antenna, $\left|B_{S}\right|=1 . h_{j k}=g_{j k} * \sqrt{\Omega_{j k}}$ and $\Omega_{j k}=S_{j k} L_{j k}$, in which $h_{j k}$ is the channel gain from transmitting antenna $j$ to user $k, g_{j k}$ is the small scale channel fading and $S_{j k}$ represents shadow fading and $L_{j k}$ represents path loss. We only consider path loss as the large scale channel fading in this paper. The receiving Signal-to-Interference-plusNoise-Ratio (SINR) for user $k$ is:

$$
\gamma_{k}=\frac{\sum_{j \in B_{S}} P_{t}\left|h_{j k}\right|^{2}}{\sum_{j \in B_{I}} P_{t}\left|h_{i k^{\prime}}\right|^{2}+P_{n}}
$$

The transmitting SNR is $\gamma_{0}=\frac{P_{t}}{P_{n}}$, where $P_{n}=N_{0} \times B W$ is the noise power and $B W$ is the bandwidth.

Thus, the downlink capacity is

$$
C_{k}=B W_{k} \times \log _{2}\left(1+\gamma_{k}\right)
$$

where $B W_{k}$ is the bandwidth of user $k$. The ergodic Shannon capacity for users in one cell at a certain time can be expressed by:

$$
E\left[C_{k}\right]=B W_{k} \int_{0}^{\infty} \log _{2}(1+\gamma) f_{\Gamma}(\gamma) d \gamma
$$

for $k=1, \ldots, N_{u e}$, where $N_{u e}$ is the total number of user equipments. $f_{\Gamma}(\gamma)$ is the probability density function (PDF) of the receiving SINR.

\section{Analytical Transmitting SNR Threshold}

\section{A. Problem Formulation}

We use the downlink ergodic capacity as the target of the decision on whether to use CJT or non-CJT. If the ergodic capacity of CJT $C_{C}$ is greater than the ergodic capacity of non-CJT $C_{N}$, i.e. $E\left[C_{C}\right]>E\left[C_{N}\right]$, CJT should be used to strengthen the performance, and otherwise, we do not have to use CJT. Non-CJT can save the total power consumption compared with that of CJT because one user is supported by one antenna with transmitting power $P_{t}$ when no CJT is used, while one user is supported by multiple antennas both with transmitting power $P_{t}$ in the CJT scenario. Thus Non-CJT should be selected as long as it can provide receiving SINR that is higher than or euqual to CJT. The problem is defined to find $\gamma_{t h}$, the threshold of transmitting SNR as:

$$
\mathcal{P}:\left\{\begin{array}{l}
\text { find } \gamma_{t h} \\
\text { for } \forall \gamma_{0} \leq \gamma_{t h}, \\
\text { for } \forall \gamma_{0}>\gamma_{t h}, \quad \text { s.t. } E\left[C_{C} \mid \gamma_{0}\right] \geq E\left[C_{C} \mid \gamma_{0}\right]<E\left[C_{N} \mid \gamma_{0}\right]
\end{array}\right.
$$

For a fair comparison, the capacity is evaluated under the same bandwidth, FFR pattern and channel fading condition. We consider the receiving SINR as a function of the transmitting SNR $\gamma_{0}$. The value of transmitting SNR will affect the receiving SINR and the capacity, depending on the linear property, the constraints of the problem can be turned into the comparison between receiving SINR as:

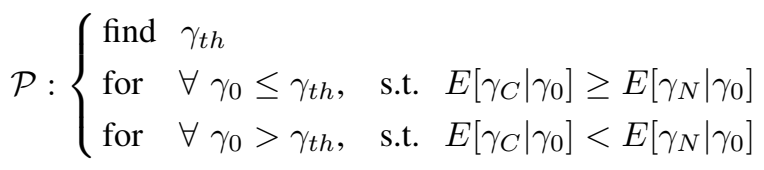

where $\gamma_{C}$ is the receiving SINR when using CJT and $\gamma_{N}$ is the receiving SINR when using non-CJT following (2). We 
assume the expectation of the small scale fading channel gain is constant $E\left[\left|g_{i k^{\prime}}\right|^{2}\right]=G$, then we get:

$$
\begin{aligned}
& E\left[\gamma_{k} \mid \gamma_{0}\right]=E\left[\frac{\sum_{j \in B_{S}} P_{t}\left|h_{j k}\right|^{2}}{\sum_{i \in B_{I}} P_{t}\left|h_{i k^{\prime}}\right|^{2}+P_{n}}\right] \\
& \approx \frac{\sum_{j \in B_{S}} P_{t} E\left[\left|g_{j k}\right|^{2} L_{j}\right]}{\sum_{i \in B_{I}} P_{t} E\left[\left|g_{i k^{\prime}}\right|^{2} L_{i}\right]+P_{n}}=\frac{\sum_{j \in B_{S}} E\left[L_{j}\right]}{\sum_{i \in B_{I}} E\left[L_{i}\right]+\hat{\gamma}_{0}}{ }^{-1}
\end{aligned}
$$

where $\hat{\gamma_{0}}=G \gamma_{0}$. We use the Jensen's inequality [9] to get an approximation of (7). Path loss is considered as the only factor in large-scale fading in this paper. We use the classical wireless path loss model proposed by Goldsmith in [10] given as $L_{i}=K\left(\frac{d_{i}}{d_{0}}\right)^{-\beta}$, where $K$ is a constant parameter related to the antenna features and channel attenuation, $d_{i}$ is the distance between antenna $i$ and users, $d_{0}$ is the reference distance and $\beta$ is the path loss exponent, which is a fixed value under a certain configuration. Substituting (8) into (6), we obtain:

$$
\begin{aligned}
& \frac{\left|B_{S}\right| E\left[L_{j}\right]}{\sum_{i \in B_{I}^{C}} E\left[L_{i}\right]+\hat{\gamma}_{0}^{-1}} \geq \frac{E\left[L_{j}\right]}{\sum_{i \in B_{I}^{N}} E\left[L_{i}\right]+\hat{\gamma}_{0}^{-1}} \\
& \hat{\gamma}^{-1} \geq \sum_{i \in B_{I t 1}^{C}} E\left[L_{i}\right]+\sum_{i \in B_{I t 2}^{C}} E\left[L_{i}\right]-\left|B_{S}\right| \sum_{i \in B_{I t 2}^{N}} E\left[L_{i}\right]
\end{aligned}
$$

where $B_{I}^{C}$ and $B_{I}^{N}$ are the sets of interfering transmitting antennas in CJT and non-CJT cases respectively. $B_{\text {Iti }}^{C}$ denotes the interfering transmitting antennas set in tier $i, i=1,2,3$. In CJT case, no co-ordination between neighbouring base stations, thus interference exists in both tier one and tier two, given as : $B_{I}^{C}=B_{I t 1}^{C} \cup B_{I t 2}^{C}$. However, in non-CJT case, there is no interfering basestation in tier one $B_{I t 1}^{N}=\emptyset$, thus the total interference of non-CJT case is given as $B_{I}^{N}=B_{I t 2}^{N}$.

\section{B. Expectation of Distance between cell edge users and inter- fering base stations}

According to [11], if the mobile users are independently and uniformly distributed in their respective cells, the PDF of the mobile users' locations polar coordinates $(r, \theta)$ relative to the most closest base station are:

$$
\begin{cases}f_{r}(r)=\frac{2\left(r-R_{0}\right)}{\left(R-R_{0}\right)^{2}}, & R_{0} \leq r \leq R, \\ f_{\theta}(\theta)=\frac{1}{2 \pi}, & 0 \leq \theta \leq 2 \pi\end{cases}
$$

where $R$ is cell radius and $R_{0}$ is cell center radius. The distance between mobile users to the interfering cell base station is

$$
d(r)=\sqrt{r^{2}+2 r D \sin (\theta)+D^{2}}
$$

where $D$ is the distance between the mobile user's respective cell base station and its interfering base station.

The expectation of the interfering distance is:

$$
\begin{aligned}
& E[d]=\int_{0}^{2 \pi} \int_{R_{0}}^{R} d(r) f_{r}(r) f_{\theta}(\theta) d r d \theta \\
& =\frac{2 \int_{0}^{2 \pi} \int_{R_{0}}^{R} \sqrt{r^{2}+2 r D \sin (\theta)+D^{2}}\left(r-R_{0}\right) d r d \theta}{2 \pi\left(R-R_{0}\right)^{2}} .
\end{aligned}
$$

Because there is no explicit solution for (11), we deduce it using an approximations for tier one and tier two. For tier one where $D=\sqrt{3} R$ and $R_{0}=2 / 3 R$, we use the following approximation:

$$
\begin{gathered}
\int_{0}^{2 \pi} \int_{R_{0}}^{R} \sqrt{r^{2}+2 \sqrt{3} R r \sin (\theta)+3 R^{2}} d r d \theta \\
\leq 2 \pi \int_{R_{0}}^{R} \sqrt{r^{2}+2 \sqrt{2} R r+2 R^{2}} d r .
\end{gathered}
$$

Hence, the resulting approximation for the expectation of the interfering distance is $D=2 \sqrt{3} R$ and $R_{0}=2 / 3 R$ :

$$
E[d] \leq \frac{2 \int_{R_{0}}^{R}(r+\sqrt{2} r)\left(r-R_{0}\right) d r}{\left(R-R_{0}\right)^{2}}=\left(\frac{5}{9}+\sqrt{2}\right) R
$$

We use the following approximation for tier two where :

$$
\begin{array}{r}
\int_{0}^{2 \pi} \int_{R_{0}}^{R} \sqrt{r^{2}+4 \sqrt{3} R r \sin (\theta)+12 R^{2}} d r d \theta \\
\leq 2 \pi \int_{R_{0}}^{R} \sqrt{r^{2}+4 \sqrt{2} R r+8 R^{2}} d r
\end{array}
$$

Hence, the resulting approximation for the expectation of the interfering distance is:

$$
E[d] \leq \frac{2 \int_{R_{0}}^{R}(r+2 \sqrt{2} r)\left(r-R_{0}\right) d r}{\left(R-R_{0}\right)^{2}}=\left(\frac{8}{9}+2 \sqrt{2}\right) R
$$

Since we have used the approximation in the calculating of the expectation of the interfering distance, we have also checked difference between the original expectation and the approximated one. The mean squared error (MSE) between (11) and (13) is $-27.87 \mathrm{~dB}$ for tier one and $-29.72 \mathrm{~dB}$ for tier two.

\section{Decision Making on CJT or Non-CJT}

Substituting (13) and (15) into (9), we obtain the threshold for the transmitting SNR :

$$
\gamma_{t h}=\frac{\left(R / d_{0}\right)^{\beta}(G K)^{-1}}{\left(\left(\left|B_{I t 1}^{C}\right|\right) A_{1}^{-\beta}+\left(\left|B_{I t 2}^{C}\right|-\left|B_{S}\right|\left|B_{I t 2}^{N}\right|\right) A_{2}^{-\beta}\right)}
$$

where $A_{1}=5 / 9+\sqrt{2}$ and $A_{2}=8 / 9+2 \sqrt{2}$ from (13) and (15) respectively.

Therefore, our decision making on whether to use CJT or not is: For a given transmitting SNR $\gamma_{0}$, if $\gamma_{0}<\gamma_{t h}$, CJT should be used to obtain higher capacity; else if $\gamma_{0} \geq \gamma_{t h}$, non-CJT can obtain higher capacity and also save the total power consumption compared with that of CJT.

For a limited size cellular network, the parameters in (16) are all positive real and far less than infinity, then the threshold of the transmitting SNR is positive real and far less than infinity, $0<\gamma_{t h} \ll+\infty$. Thus, the transmitting SNR is an important factor in analysis of CJT in FFR-aided DAS and can not be assumed to be positive infinite. Thus the scenarios in previous literature [5][6] that assume noise is zero or small enough to be ignored are not suitable for the analysis of CJT 


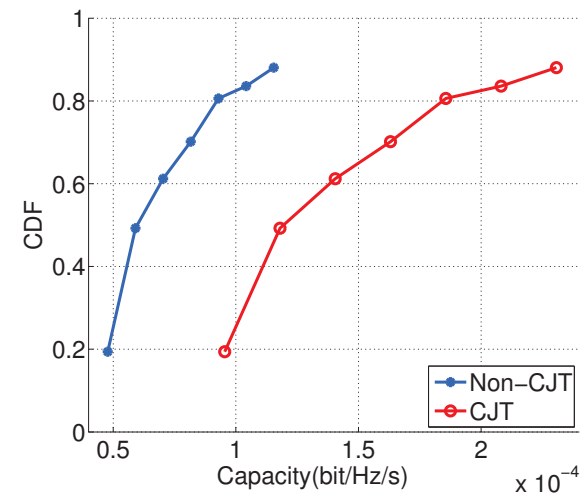

(a) Capacity CDF in low SNR condition

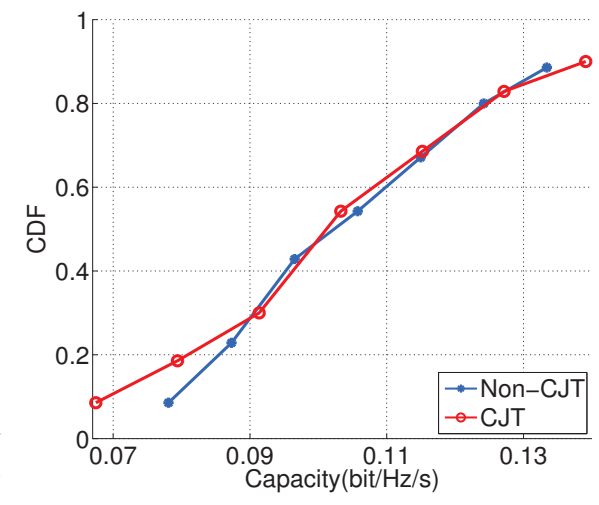

(b) Capacity CDF in threshold SNR condition

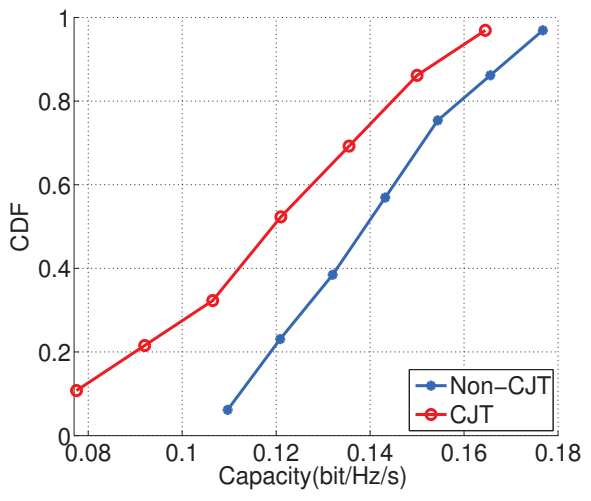

(c) Capacity CDF in high SNR condition

Fig. 2: Comparison of capacity CDF under three different transmitting SNR values, $B_{S}=2$.

in FFR-aided DAS. In this paper we select a classic FFR pattern. For other possible FFR patterns, considering the same transmission schemes, a more general expression can be given as:

$$
\gamma_{t h}=\frac{\left(R / d_{0}\right)^{\beta}(G K)^{-1}}{\left(\left(\left|B_{I t 1}^{C}\right|-2\left|B_{I t 1}^{N}\right|\right) A_{1}^{-\beta}+\left(\left|B_{I t 2}^{C}\right|-\left|B_{S}\right|\left|B_{I t 2}^{N}\right|\right) A_{2}^{-\beta}\right)}
$$

where we assume

$$
\begin{aligned}
& \left(\left|B_{I t 1}^{C}\right|-\left|B_{S}\right|\left|B_{I t 1}^{N}\right|\right) A_{1}^{-\beta} \\
& \quad+\left(\left|B_{I t 2}^{C}\right|-\left|B_{S}\right|\left|B_{I t 2}^{N}\right|\right) A_{2}^{-\beta}>0 .
\end{aligned}
$$

\section{NumericAl Results AND Discussion}

The scenario considered in this paper is the cellular network C-RAN, path loss reference distance $d_{0}=100 \mathrm{~m}$ is configured in the simulation [10]. Each cell has 120 random uniformly distributed single-antenna users. For CJT case: $\left|B_{S}\right|=2$, $\left|B_{I t 1}^{C}\right|=2,\left|B_{I t 2}^{C}\right|=9$ or $\left|B_{S}\right|=3,\left|B_{I t 1}^{C}\right|=4,\left|B_{I t 2}^{C}\right|=12$; for non-CJT case: $\left|B_{S}\right|=1,\left|B_{I t 1}^{N}\right|=0,\left|B_{I t 2}^{N}\right|=6$.

\section{A. Capacity $C D F$}

Firstly, we simulate the ergodic capacity for the cell edge users with the cell radius $R=500 \mathrm{~m}$ and two co-ordinated basestations $B_{S}=2$ to illustrate the effect of the transmitting SNR on the capacity. Three transmitting SNR values $\left(\gamma_{0}<\gamma_{t h}, \gamma_{0}=\gamma_{t h}\right.$ and $\left.\gamma_{0}>\gamma_{t h}\right)$ have been picked for illustration. The Cumulative Distribution Function (CDF) of downlink ergodic capacity is evaluated using Monte-Carlo Simulation. The results are shown in Fig. 2 which match our analysis in (16). These results verify our analysis on the existence of the transmitting SNR threshold for the decision making of using CJT or non-CJT for all cell edge users and also show that the transmitting threshold is far less than infinite positive thus the transmitting SNR should be considered as a key factor in the research on CJT in FFR-aided DAS.

\section{B. Receiving SINR}

Secondly, we simulate the average receiving SINR with various transmitting SNR and two cell radius settings $R=300 \mathrm{~m}$ and $R=500 \mathrm{~m}$. We use the differential receiving SINR as the illustration. The differential receiving SINR is given as:

$$
\Delta \gamma=E\left[\gamma_{C} \mid \gamma_{0}\right]-E\left[\gamma_{N} \mid \gamma_{0}\right]
$$

where $E\left[\gamma_{C} \mid \gamma_{0}\right]$ and $E\left[\gamma_{N} \mid \gamma_{0}\right]$ follow (7) for CJT and nonCJT configurations respectively.

According to our analytical result in (16), if $\gamma_{0}<\gamma_{t h}$, we have $\Delta \gamma>0$, which means CJT provides better performance in lower SNR conditions; or if $\gamma_{0}>\gamma_{t h}$, we have $\Delta \gamma<0$, which means non-CJT can provide better performance in high SNR conditions. The threshold will happen when $\gamma_{0}=\gamma_{t h}$ and $\Delta \gamma=0$.

The simulation results in Fig. 3 show the cross point of nonCJT and CJT differential receiving SINR curves with $B_{S}=2$ and $B_{S}=3$ respectively. Our analytical results on the SNR threshold obtained from (16) are also marked as dashed lines in the figures. The value of transmitting SNR at the intersection of the curves with $\Delta \gamma=0$ is slightly less than the SNR threshold obtained from (16). This also follows our analytical result, because we have used an upper bounded approximation.

Consider the thermal noise power density $N_{0}=k T_{0}$ [10] which has been chosen in the simulation setting of most Stateof-the-Art in this area, where $k$ is the Boltzmann's constant $k=1.38 \times 10^{-23} \mathrm{~W} / \mathrm{K} / \mathrm{Hz}$ and $T_{0}=290 \mathrm{~K}$. Thus the thermal noise power density is $N_{0}=-174 \mathrm{dBm} / \mathrm{Hz}$, which is the value in the simulation of [5] and [6]). Assuming the transmitting power is $P_{t}=43 \mathrm{dBm}$ for $5 \mathrm{MHz}$ bandwidth, the transmitting SNR is $\gamma_{0}=120 \mathrm{~dB}$ which is greater than the transmitting SNR threshold calculated from (16). If we only consider the thermal noise, we do not have to use CJT to enhance the performance. However, if we consider other source such as the signals from other networks nearby operating in the same spectrum e.g. femto-cell, the transmitting SNR may be less than the SNR threshold, and then CJT should be used. 


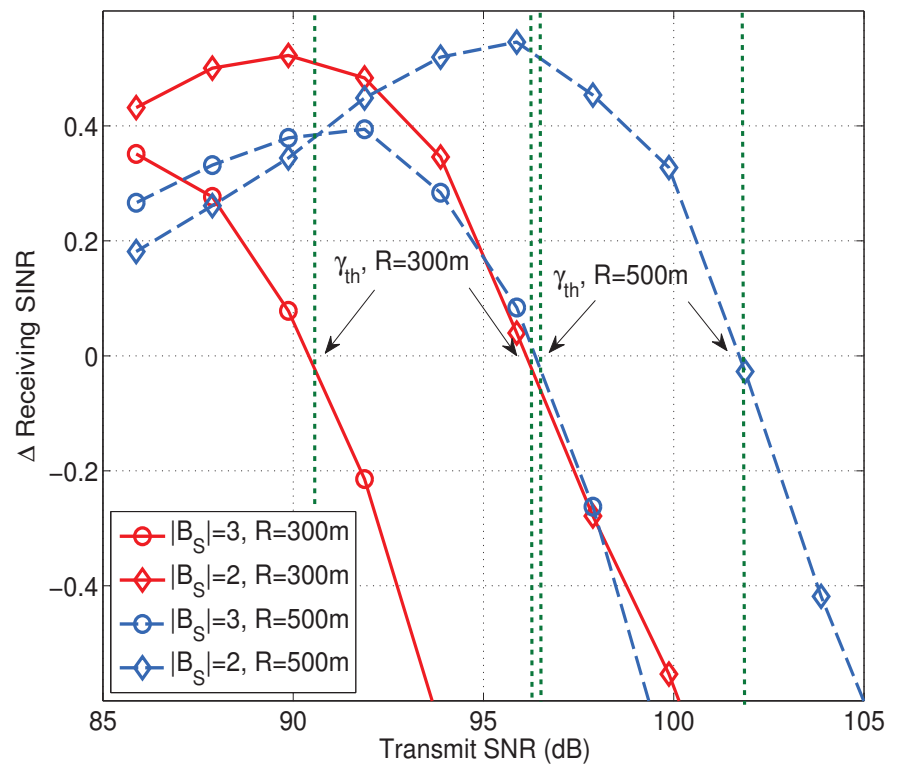

Fig. 3: Differential Receiving SINR as a function of transmitting SNR.

\section{Threshold Accuracy Evaluation}

Thirdly, we evaluate the accuracy of our threshold expression in (16) under different cell radius settings from $R=300 \mathrm{~m}$ to $R=500 \mathrm{~m}$ and different path loss exponent $\beta=2.5,3.25$ and 4 with two co-ordinated basestation $B_{S}=2$, as illustrated in Fig. 4. The MSE between our threshold and the Monte-Carlo simulation is approximately $-50.27 \mathrm{~dB}$. Thus the threshold obtained from (16) is a tight upper bound and from the figure we can observe that it is more accurate for a larger cell radius.

The threshold increases with the increase of the cell radius and the increase of the path loss exponent as illustrated in Fig. 4. Because in larger cells or greater path loss, cell edge users will be further away from the transmitting antennas or experience deeper large scale fading, thus they need CJT to obtain more capacity. Moreover, larger cells or deeper path loss will decrease the inter-cell interference caused by CJT.

\section{CONCLusion}

In this paper, we have studied the capacity of Distributed Antanna Systems for Cloud Radio Access Networks under a combination of the Fractional Frequency Reuse and Coordinated Joint Transmission between base stations. We have derived a new threshold for the transmitting SNR to decide whether to use Coordinated Joint Transmission or not in FFR-aided DAS. Moreover, we have shown that the transmitting SNR is a key factor in the analysis of FFR-aided DAS for both static and dynamically changing network topologies. Monte-Carlo simulations have been also also carried out and the results match to our analysis. The analytical expressions can be used as a guide for future wireless communication networks, such as C-RAN, without the need to carry out simulations.

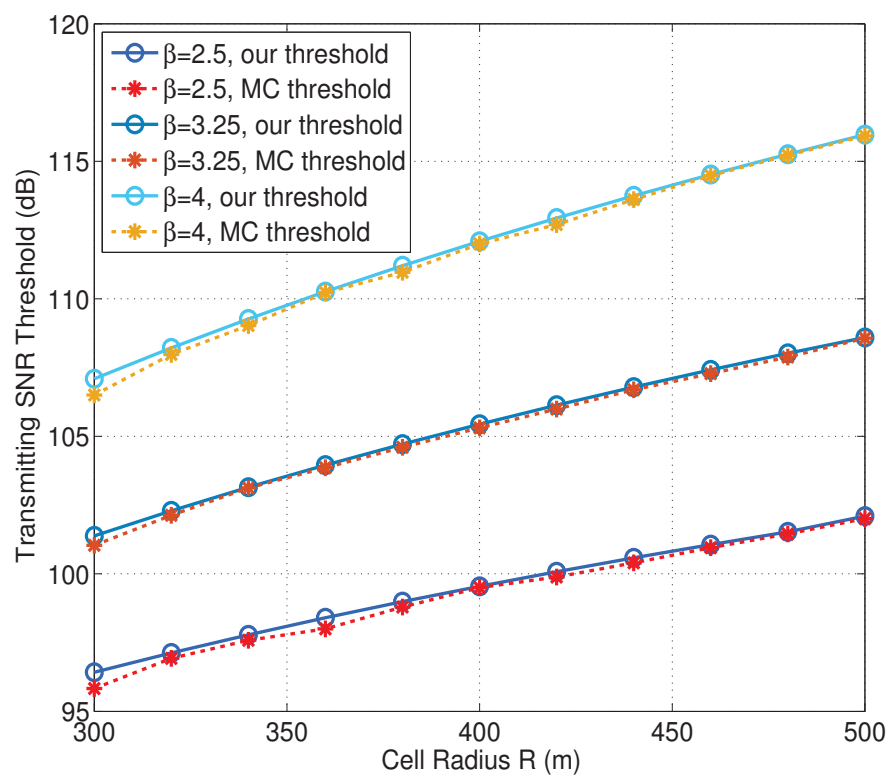

Fig. 4: Transmitting SNR threshold as a function of cell radius, $B_{S}=$ 2 .

\section{ACKNOWLEDGEMENT}

Ying $\mathrm{He}$ is a recipient of a Macquarie University Research Excellence Scholarship. This work has been supported in part by Intel's University Research Office.

\section{REFERENCES}

[1] A. M. Saleh, A. J. Rustako, and R. S. Roman, "Distributed antenna for indoor radio communications," IEEE Trans. Commun., vol. 35, no. 12, pp. 1245-1251, Dec. 1987.

[2] L. Dai, S. Zhou, and Y. Yao, "Capacity analysis in CDMA distributed antenna systems," IEEE Trans. Wireless Commun., vol. 4, no. 6, pp. 2613-2620, Nov. 2005.

[3] J. Joung, Y. K. Chia, and S. Sun, "Energy-efficient, large-scale distributed-antenna system (L-DAS) for multiple users," IEEE Journal of Selected Topics in Signal Processing, vol. 8, pp. 954-965, 2014.

[4] S. Bhaumik, S. Chandrabose, M. Jataprolu, G.Kumar, A. Muralidhar, P. Polakos, V. Srinivasan, and T. Woo, "Cloudiq: A framework for processing base stations in a data center," in Mobicom, 2012, pp. 125136.

[5] J. Hwang, S. M. Yu, S.-L. Kim, and R. Jantti, "On the frequency allocation for coordinated multi-point joint transmission," in IEEE VTC Spring, 2012, pp. 1-5.

[6] X. Zhang, Y. Sun, X. Chen, S. Zhou, J. Wang, and N. B. Shroff, "Distributed power allocation for coordinated multipoint transmissions in distributed antenna systems," IEEE Trans. Wireless Commun., vol. 12, no. 5, pp. 2281-2291, 2013.

[7] Y. He, E. Dutkiewicz, G. Fang, and J. Shi, "Differential capacity bounds for distributed antenna systems under low snr conditions," in 2014 International Symposium on Wireless Personal Multimedia Communications (WPMC), 2014, pp. 242-428.

[8] Y. He, E. Dutkiewicz, G. Fang, and M. Mueck, "Fractional frequency reuse in distributed antenna systems in cloud radio access networks," accepted by ICC Workshop on Cooperative and Cognitive Networks 2015, 2015.

[9] D. Chandler, "Introduction to modern statistical mechanics," Oxford University Press, 1987.

[10] A. Goldsmith, Wireless communications. Cambridge university press, 2005.

[11] M.-S. Alouini and A. J. Goldsmith, "Area spectral efficiency of cellular mobile radio systems," IEEE Trans. Vehicular Tech., vol. 48, no. 4, pp. 1047-1066, 1999. 